\title{
Surgical resection improves long-term survival of patients with hepatocellular carcinoma across different Barcelona Clinic Liver Cancer stages
}

This article was published in the following Dove Press journal:

Cancer Management and Research

\author{
Hui Guo ${ }^{1,2}$ \\ Tao $\mathrm{Wu}^{2}$ \\ Qiang Lu' ${ }^{1,3}$ \\ Miaojing $\mathrm{Li}^{2}$ \\ Jing-Yue Guo ${ }^{2}$ \\ Yuan Shen ${ }^{4}$ \\ Zheng $\mathrm{Wu}^{1,3}$ \\ Ke-Jun $\mathrm{Nan}^{2}$ \\ Yi Lv' ${ }^{1,3}$ \\ Xu-Feng Zhang ${ }^{1,3}$ \\ 'Department of Hepatobiliary Surgery, \\ The First Affiliated Hospital of Xi'an \\ Jiaotong University, Xi'an, Shaanxi, \\ China; ${ }^{2}$ Department of Oncology, \\ The First Affiliated Hospital of Xi'an \\ Jiaotong University, Xi'an, Shaanxi, \\ China; ${ }^{3}$ Institute of Advanced Surgical \\ Technology and Engineering, The First \\ Affiliated Hospital of Xi'an Jiaotong \\ University, Xi'an, Shaanxi, China; \\ ${ }^{4}$ Department of Epidemiology and \\ Health Statistics, School of Public \\ Health, Xi'an Jiaotong University \\ Health Science Center, Xi'an, Shaanxi, \\ China
}

Correspondence: Xu-Feng Zhang Department of Hepatobiliary Surgery, The First Affiliated Hospital of Xi'an Jiaotong University, 277 West Yanta Road, Xi'an, China

$\mathrm{Tel}+862985323626$

Email xfzhang125@xjtu.edu.cn
Objectives: Surgical resection remains a controversial treatment for hepatocellular carcinoma (HCC) within different Barcelona Clinic Liver Cancer (BCLC) stages. The objective of this study was to evaluate the long-term outcome of patients undergoing surgical resection (SR) compared to non-surgical treatments across different BCLC stages.

Patients and methods: One thousand four hundred forty-three HCC patients within BCLC 0, A, B and C stages were identified. Overall survival was compared by log-rank test among patients within different BCLC stages and among patients receiving different treatments (SR vs locoregional therapy [LRT] vs best supportive care). Propensity score matching analysis was introduced to mitigate the confounding biases between the groups.

Results: The median survival time of the patients diminished from early, intermediate to advanced BCLC stages (BCLC 0-A 43 [range 0-100] months vs BCLC B 32 [range 0-100] months vs BCLC C 27 [range 0-90] months, all $p<0.05$ ). Patients undergoing SR presented with better liver function and more favorable tumor status and, consequently, displayed significant better overall survival than patients receiving LRT or best supportive care at different BCLC stages. In adjusted cohort after propensity score matching, patients who were surgically treated consistently had more favorable outcome than those who were non-curatively treated across different BCLC stages (median survival [range]: BCLC stage B: resection 45 [0-100] months vs LRT 36 [0-81] months, $p=0.002$; BCLC stage C: resection 39 [3-77] months vs LRT 27 [0-54] months, $p=0.003$ ).

Conclusion: Surgical resection should be considered as a radical treatment for selected HCC patients regardless of the BCLC stages when appropriate.

Keywords: hepatocellular carcinoma, surgery, Barcelona Clinic Liver Cancer stages, outcome

\section{Introduction}

Several staging systems have been developed for prognosis evaluation of hepatocellular carcinoma (HCC) after treatments. ${ }^{1}$ The Barcelona Clinic Liver Cancer (BCLC) system is the most widely used scheme, since it is the only staging system incorporating severity of liver cirrhosis, tumor burden and patient performance status, with the advantage of prognosis assessment and treatment allocation. ${ }^{1-4}$ BCLC is now endorsed by the American Association for the Study of Liver Disease and the European Association for the Study of Liver. ${ }^{2,3}$ According to the BCLC staging system, patients with early stages (stage 0 and $\mathrm{A}$ ) are recommended for surgical resection, while patients with intermediate (stage B) and advanced (stage C) HCC are recommended for transcatheter arterial chemoembolization (TACE) and sorafenib. However, the major limits of 
BCLC system are the great prognostic heterogeneity and the distinct tumor condition of patients within each stage, especially in intermediate and advanced stages. ${ }^{5,6}$ Recently, some studies, mostly from Asian countries that are endemic areas of $\mathrm{HCC}$, have focused on expending the indication of surgical resection in intermediate and advanced HCC, and demonstrated improved survival by surgical resection over other palliative treatments. ${ }^{6-10}$ However, some other studies found equivalent effectiveness of TACE to surgical resection among operable HCC in intermediate and advanced HCC. ${ }^{11}$

There is still no strong evidence of the benefits of surgical resection over palliative treatments across different BCLC stages. In this study, we aimed at evaluating the potential benefits of patients undergoing surgical resection vs nonsurgical treatments in a large cohort of HCC patients within different BCLC stages.

\section{Patients and methods}

\section{Patients}

Patients diagnosed with HCC and admitted for treatment of HCC from January 2008 to December 2013 were retrospectively reviewed based on the patient database of the First Affiliated Hospital of Xi' an Jiaotong University, Xi'an, China. The primary treatments were divided into three major groups. Surgical resection was defined as hepatic resection for $\mathrm{HCC}$, irrespective of whether or not the patients have received locoregional therapies (LRTs), and the survival time was calculated from the time of surgery. LRTs were defined as non-radical treatments including radiofrequency ablation (RFA), TACE, hepatic artery or portal vein infusion, radiotherapy and so on. The patients were followed since the first time of administering LRT. However, patients with BCLC stage 0-A receiving RFA were considered as getting curative treatment and were analyzed separately from the LRT group. The rest of the patients receiving noninvasive treatments, but only supportive care were considered in the best supportive care (BSC) group.

Demographic characteristics, lifestyle factors, clinical and pathologic data were collected at admission, including age, gender, education background, smoking, alcohol abuse, regular screening, diabetes, hepatitis status, liver cirrhosis, liver function, alpha-fetoprotein level, tumor number and size, and vascular invasion. Patients with hepatitis B virus (HBV) and/or hepatitis $\mathrm{C}$ virus (HCV) were recommended for a routine screening of liver function, $\mathrm{HBV} / \mathrm{HCV}$ copies, alphafetoprotein and ultrasound examination once or twice per year. Tumor number, size and vascular invasion were mainly documented based on radiologic examination, and then confirmed by surgical or pathologic examination if appropriate. Liver cirrhosis was diagnosed based on the pathologic examination as presence of regenerating nodules of hepatocytes and fibrosis, and/or evaluated by ultrasound or computed tomography showing a small and nodular liver along with enlarged caudate lobe, widening of the liver fissures and so on. ${ }^{12,13}$

BCLC classification was used as the standard staging system in patient stratification. ${ }^{4}$ However, we included patients with single large tumor $(>5 \mathrm{~cm})$ as BCLC stage B based on some comprehensive studies, since tumor size was significantly correlated with prognosis. ${ }^{14,15}$ The study was approved by the Ethics Committee of The First Affiliated Hospital of Xi'an Jiaotong University, which waived informed consent, since the data were analyzed from the electronic medical record and reported without personal identifiers.

\section{Statistical analysis}

Numerical data were expressed as median and range. Categorical data were described by frequency and percentages. Numerical data were compared among the groups with Student's $t$-test or Wilcoxon rank sum test. Categorical data were compared by using chi-square test or Fisher's exact test. The survival curves were plotted by Kaplan-Meier method, and the survival rates among different groups were compared by the log-rank test.

To eliminate selection bias, we introduced propensity score matching (PSM) analysis in this study to balance the baseline differences of the patients undergoing surgical resection and LRT. Propensity score analysis with 1:1 matching was performed to generate matched pairs of patients. All $p$ values were derived from two-tailed tests, and $p$ value $<0.05$ was considered statistically significant. Statistical analysis was carried out using SPSS 22.0 (IBM Corporation, Armonk, NY, USA).

\section{Results}

A total of 2060 patients with HCC were identified; 310 $(15 \%)$ patients with inadequate information were excluded from the study. Also, 265 patients with BCLC stage D, 30 patients receiving liver transplant and 12 patients treated with sorafenib were also excluded. Finally, 1443 patients were included (Figure 1).

\section{Survival of patients in different BCLC stages}

Among the 1443 patients, there were 330 (22.9\%) patients in BCLC stage 0-A, 798 (55.3\%) in BCLC stage B and 315 $(21.8 \%)$ in BCLC stage $\mathrm{C}$ (Figure 1). Surgical resection was performed in $422(29.2 \%)$ of the patients. The baseline 


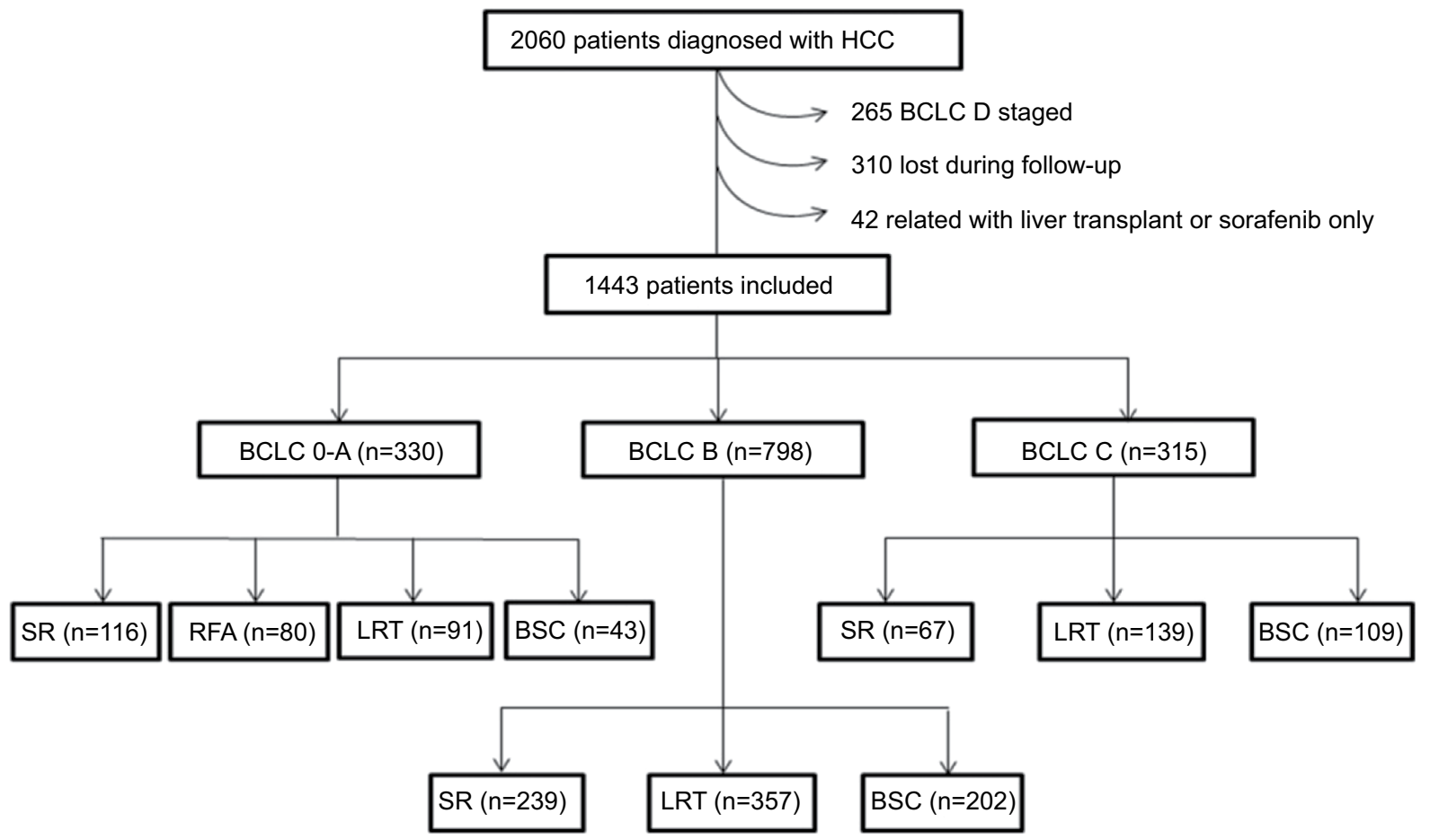

Figure I Study flowchart and inclusion of participants.

Abbreviations: BCLC, Barcelona Clinic Liver Cancer; BSC, best supportive care; HCC, hepatocellular carcinoma; LRT, locoregional therapy; RFA, radiofrequency ablation; SR, surgical resection.

characteristics of patients in the three groups are compared in Table 1. Gender and age were not different among them, but patients in BCLC C had worse living habits including smoking and alcohol abuse than the patients in the other two groups. The etiology of the underlying liver disease was not different among the three groups, with all being predominantly HBV positive. Since regular HCC screening was more likely to be performed, patients in BCLC 0-A stage were more likely to present with Child-Pugh class A and, therefore, to have a higher frequency of surgical resection than the patients in the other two groups. Of note, the 30- and 90-day mortality rates were not significantly different among patients within different BCLC stages. The median survival time was 43 (range 0-250) months in BCLC 0-A group, 32 (range 0-100) months in BCLC B group and 27 (range 0-90) months in BCLC C group. As shown in Figure 2, the 1-, 3- and 5-year survival rates were $82 \%, 53 \%$ and $28 \%$ in BCLC 0 -A group, $78 \%$, $36 \%$ and $20 \%$ in BCLC B group and $71 \%, 40 \%$ and $11 \%$ in BCLC C group ( $p<0.05$ between any two groups).

\section{Survival benefits of surgical resection in BCLC 0-A}

Among the 330 patients with BCLC stage 0-A HCC, 116 (35.2\%) underwent surgical resection, 80 (24.2\%) underwent
RFA, 91 (27.6\%) underwent TACE and 43 (13.0\%) received only BSC. Patients receiving RFA had comparable survival with those undergoing surgical resection (median survival [range]: RFA 50 [0-100] vs resection 52 [0-100] months, $p=0.932$; Figure 3 ), but significantly better survival than patients treated with TACE or BSC in BCLC stage 0-A (median survival [range]: RFA 50 [0-100] months, resection 52 [0-100] months vs TACE 32 [0-81] months, BSC 28 [0-77] months; all $p<0.001$; Figure 3 ).

\section{Survival benefits of surgical resection in BCLC stage B}

Among the 798 patients within BCLC stage B, 239 (29.9\%) patients undergoing surgical resection had significantly better survival rates than patients receiving either LRT $(n=357,44.7 \%)$ or BSC $(n=202,25.3 \%)$, with the (median survival [range]) being (resection 45 [0-100] months vs LRT 36 [0-92] months, BSC 30 [0-70] months; both $p<0.01$; Figure $4 \mathrm{~A}$ ). However, patients undergoing surgical resection were younger, had better liver function of Child-Pugh class and less bilobar lesions than those receiving LRT (all $p<0.05$, Table 2 ). To further confirm the survival benefits of surgery, we performed PSM analysis between the patients undergoing surgical resection and 
Table I Characteristics of the patients with hepatocellular carcinoma enrolled according to different BCLC stages

\begin{tabular}{|c|c|c|c|c|}
\hline Variables & $\begin{array}{l}\text { Total } \\
(\mathrm{N}=\mid \mathbf{4 4 3})\end{array}$ & $\begin{array}{l}\text { BCLC stage 0-A } \\
(n=330)\end{array}$ & $\begin{array}{l}\text { BCLC stage B } \\
(n=798)\end{array}$ & $\begin{array}{l}\text { BCLC stage } C \\
(n=3 \mid 5)\end{array}$ \\
\hline Male gender & II 70 (8I.I\%) & $265(80.3 \%)$ & $644(80.7 \%)$ & $26 \mid(82.9 \%)$ \\
\hline Age (years) & $55(7-88)$ & $54(24-82)$ & $55(13-88)$ & $53(7-87)$ \\
\hline History of cigarette smoking* & $652(45.2 \%)$ & $\mid 38(4 \mid .8 \%)$ & 347 (43.5\%) & $167(53.0 \%)$ \\
\hline History of alcohol abuse* & 499 (34.6\%) & $98(29.7 \%)$ & $271(34.0 \%)$ & $130(4 \mid .3 \%)$ \\
\hline Regular HCC screening** & $745(51.6 \%)$ & $200(60.6 \%)$ & $382(47.9 \%)$ & $163(51.7 \%)$ \\
\hline Diabetes & $119(8.2 \%)$ & $31(9.4 \%)$ & $61(7.6 \%)$ & $27(8.6 \%)$ \\
\hline HBV positivity & $1046(72.2 \%)$ & $252(76.4 \%)$ & 547 (68.5\%) & $247(78.4 \%)$ \\
\hline HCV positivity & $76(5.3 \%)$ & $26(7.9 \%)$ & 37 (4.6\%) & $13(4.1 \%)$ \\
\hline Liver cirrhosis** & 957 (66.3\%) & $242(73.3 \%)$ & $488(61.1 \%)$ & 227 (72.1\%) \\
\hline Child-Pugh class $\mathrm{B}^{* *}$ & $470(32.6 \%)$ & $65(19.7 \%)$ & $279(35.0 \%)$ & $126(40.0 \%)$ \\
\hline AFP $>200 \mathrm{ng} / \mathrm{mL}^{*}$ & $802(55.6 \%)$ & I 46 (44.2\%) & $439(55.0 \%)$ & $217(68.9 \%)$ \\
\hline Maximal diameter $\geq 5 \mathrm{~cm} * *$ & $666(46.2 \%)$ & 0 & $463(58.0 \%)$ & $204(64.8 \%)$ \\
\hline Three or more tumor nodules** & $190(13.2 \%)$ & 0 & $142(17.8 \%)$ & $48(15.2 \%)$ \\
\hline \multicolumn{5}{|l|}{ Primary treatments $* * *$} \\
\hline SR & $422(29.2 \%)$ & $116(35.2 \%)$ & 239 (29.9\%) & $67(21.3 \%)$ \\
\hline $\mathrm{LRT}^{\mathrm{a}}$ & $667(46.2 \%)$ & 91 (5I.8\%) & $357(44.7 \%)$ & $139(44.1 \%)$ \\
\hline Best supportive care & $354(24.5 \%)$ & $43(13.0 \%)$ & $202(25.3 \%)$ & $109(34.6 \%)$ \\
\hline 30-day mortality* ** & $66(4.6 \%)$ & $11(3.3 \%)$ & $34(4.3 \%)$ & $21(6.7 \%)$ \\
\hline 90-day mortality*,** & $143(9.9 \%)$ & $19(5.8 \%)$ & $75(9.4 \%)$ & $49(15.6 \%)$ \\
\hline Median survival time (months) $*, * *$ & $33(0-100)$ & $43(0-100)$ & $32(0-100)$ & $27(0-90)$ \\
\hline
\end{tabular}

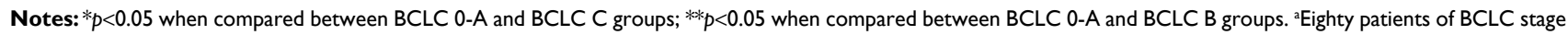
0 -A receiving radiofrequency ablation were excluded.

Abbreviations: AFP, alpha-fetoprotein; BCLC, Barcelona Clinic Liver Cancer; BSC, best supportive care; HBV, hepatitis B virus; HCC, hepatocellular carcinoma; HCV, hepatitis C virus; LRT, locoregional therapy; SR, surgical resection.

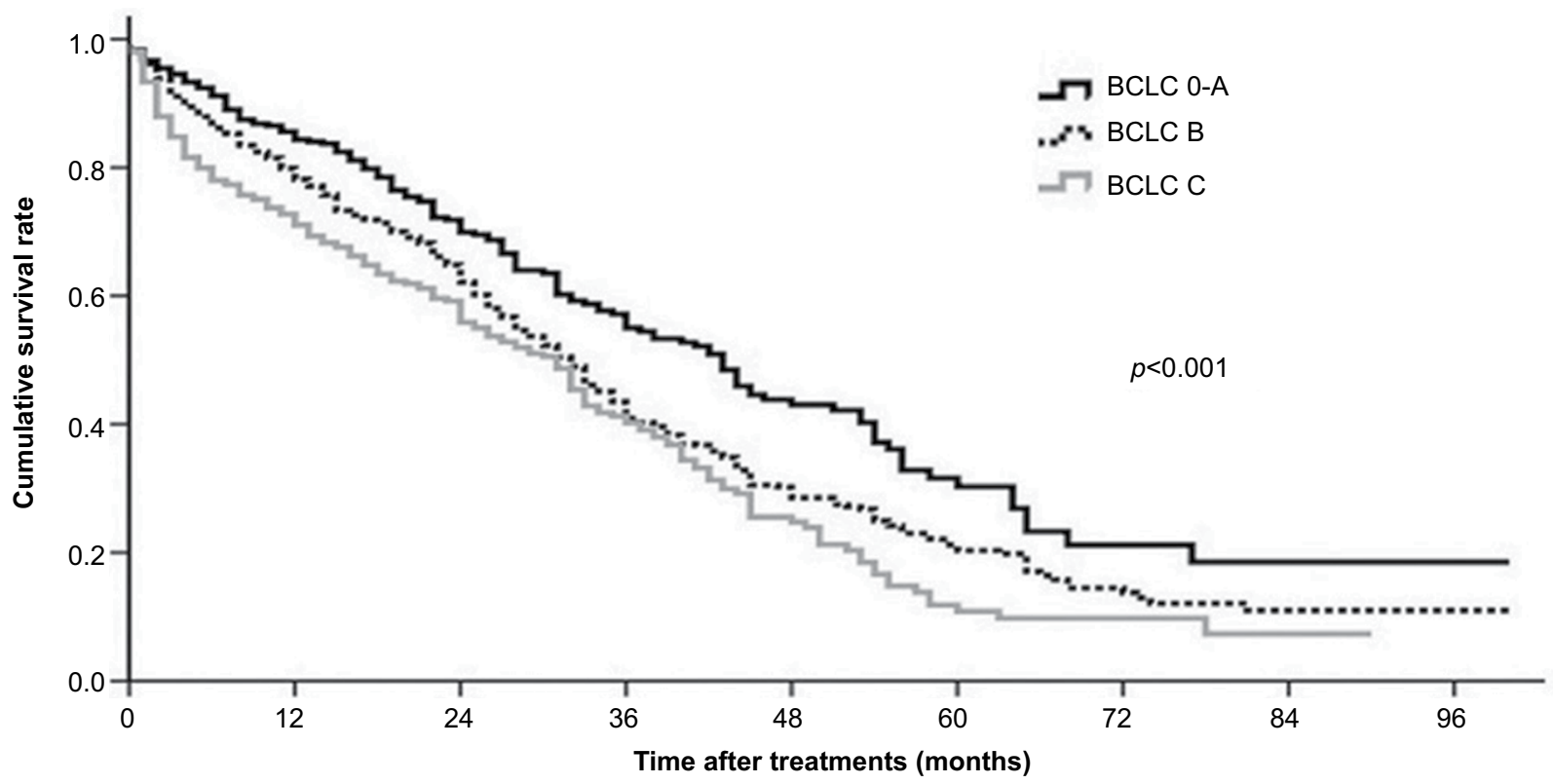

Figure 2 Kaplan-Meier survival curves of patients in different BCLC stages.

Abbreviation: BCLC, Barcelona Clinic Liver Cancer.

LRT. Exactly 238 pairs of patients in surgical resection and LRT group were selected in the matched cohort (Table 2). The patients in the two groups were well matched in age, gender, smoking, alcohol abuse, diabetes, liver cirrhosis, Child-Pugh classification, HBV/HCV status, tumor size, number and lobes involved. After PSM analysis, it was consistently identified that surgical resection showed significant survival benefits over LRT in BCLC staged B HCC (median survival [range]: 45 [0-100] vs 36 [0-81] months, $p=0.002$; Figure 4B). 


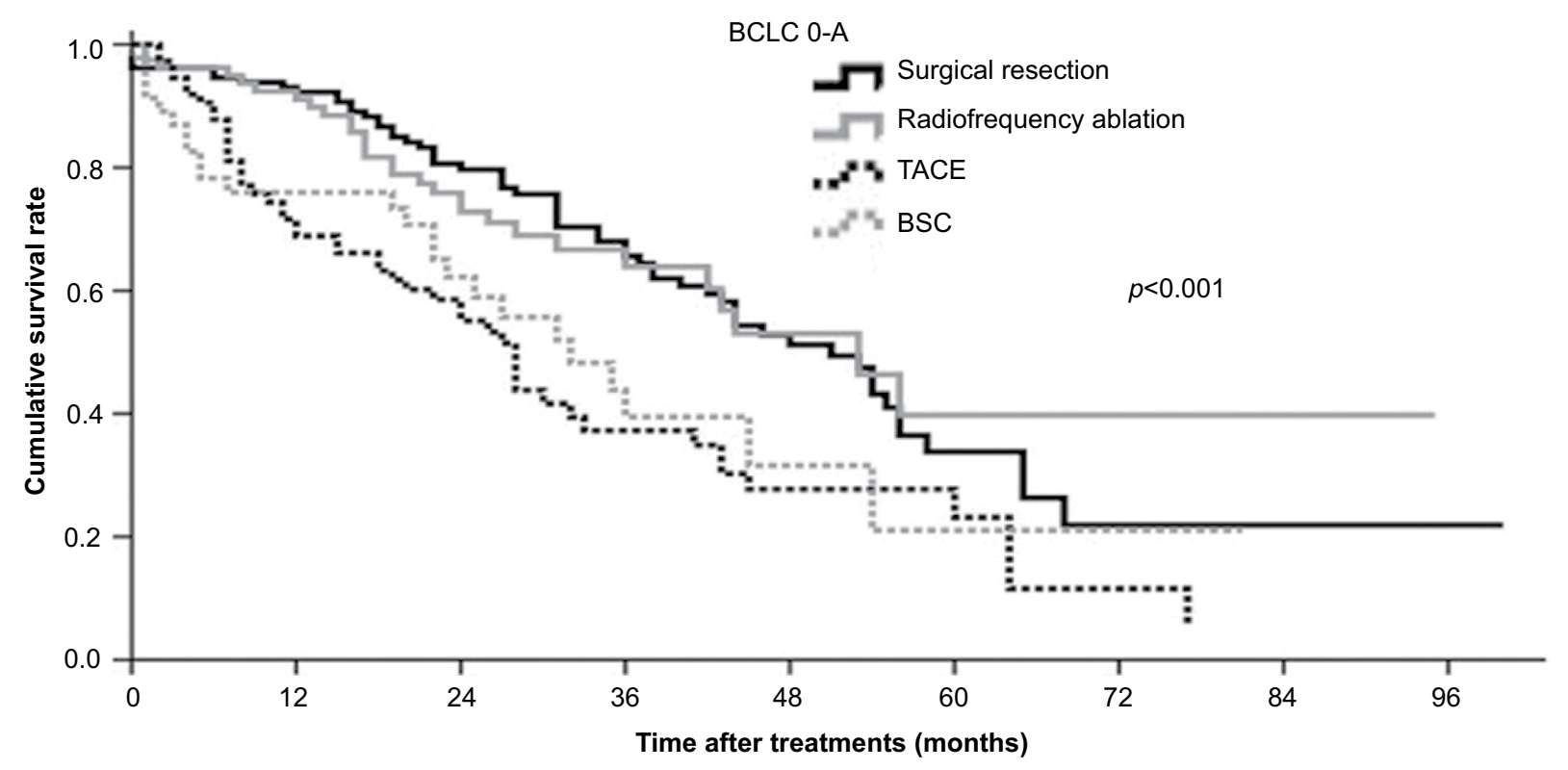

Figure 3 Overall survival of patients within BCLC stage 0-A HCC following different treatments.

Abbreviations: BCLC, Barcelona Clinic Liver Cancer; BSC, best supportive care; HCC, hepatocellular carcinoma; TACE, transcatheter arterial chemoembolization.

BCLC B
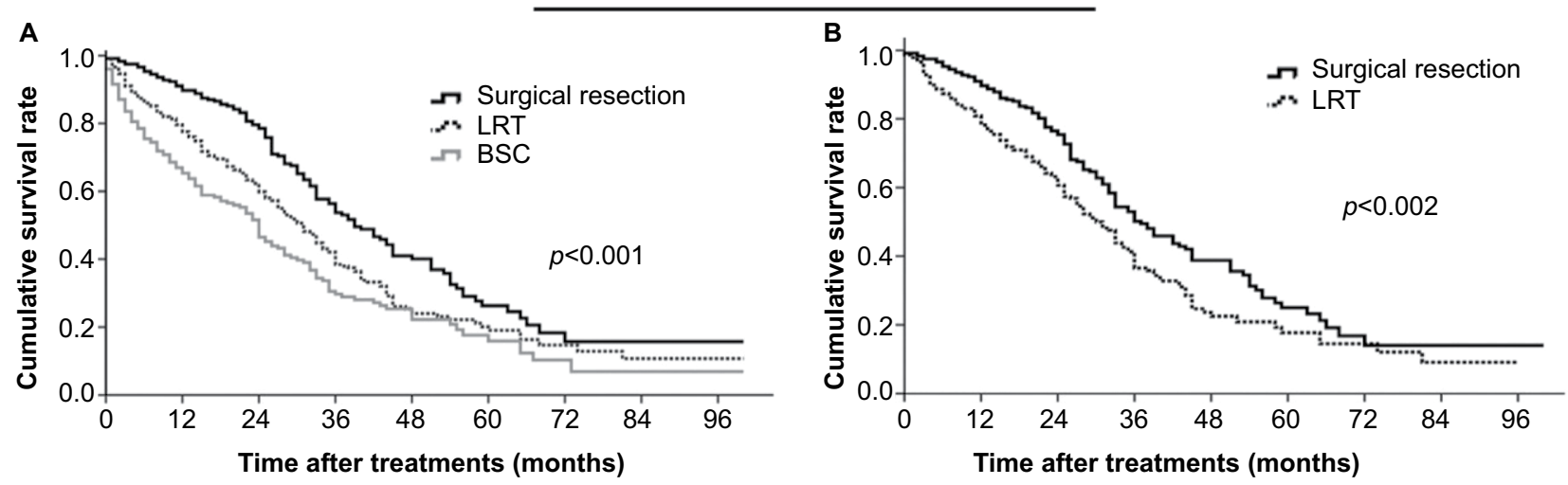

Figure 4 Overall survival of patients in BCLC stage B after different treatments in unadjusted (A) and adjusted (B) cohorts. Abbreviations: BCLC, Barcelona Clinic Liver Cancer; BSC, best supportive care; LRT, locoregional therapy.

\section{Survival benefits of surgical resection in BCLC stage C}

Among the 315 patients within BCLC stage C, 67 (21.3\%) patients underwent surgical resection and showed significant better survival rates than patients receiving either LRT $(n=139,44.1 \%)$ or BSC $(n=109,34.6 \%)$, with the (median survival [range]) being (resection 42 [1-90] vs LRT 31 [0-78] months, BSC 24.5 [0-65] months; both $p<0.01$; Figure 5A). After excluding the eight cases with extrahepatic metastasis, we had 198 patients undergoing surgical resection and LRT.
Notably, patients undergoing surgical resection had better liver function of Child-Pugh class and more favorable tumor status characterized by tumor size, number and location vs patients receiving LRT (all $p<0.05$, Table 3 ). In the propensity model, the baseline characteristics and tumor status were well matched between the 53 pairs of patients undergoing surgical resection and LRT (Table 3). Also, we further demonstrated significant survival benefits of surgical resection vs LRT (median survival [range]: 39 [3-77] vs 27 [0-54] months, $p=0.003$; Figure 5B). 
Table 2 Characteristics of SR and LRT groups within BCLC stage B before and after PSM analysis

\begin{tabular}{|c|c|c|c|c|c|c|}
\hline \multirow[t]{2}{*}{ Variables } & \multicolumn{3}{|l|}{ Before PSM } & \multicolumn{3}{|l|}{ After PSM } \\
\hline & SR $(n=239)$ & LRT $(n=357)$ & $p$-value & SR $(n=238)$ & LRT $(n=238)$ & $p$-value \\
\hline Male gender & $196(82.0 \%)$ & $289(81.0 \%)$ & 0.746 & $43(18.1 \%)$ & 49 (20.6\%) & 0.486 \\
\hline Age (years) & $53(13-78)$ & $55(17-88)$ & 0.022 & $54(13-78)$ & $55(17-88)$ & 0.102 \\
\hline Smoking & 108 (45.2\%) & $152(42.6 \%)$ & 0.529 & $107(45.0 \%)$ & $101(42.4 \%)$ & 0.579 \\
\hline Alcohol abuse & $89(37.2 \%)$ & 117 (32.8\%) & 0.292 & $88(37.0 \%)$ & $77(32.4 \%)$ & 0.289 \\
\hline Diabetes & 17 (7. I\%) & $25(7.0 \%)$ & 1.000 & 17 (7.1\%) & $16(6.7 \%)$ & 1.000 \\
\hline HBV positivity & 179 (74.9\%) & 252 (70.6\%) & 0.249 & 177 (74.4\%) & 167 (70.2\%) & 0.305 \\
\hline HCV positivity & $9(3.8 \%)$ & 17 (4.8\%) & 0.560 & $9(3.8 \%)$ & 12 (5.0\%) & 0.503 \\
\hline Liver cirrhosis & 167 (69.9\%) & $272(76.2 \%)$ & 0.086 & 166 (69.7\%) & 167 (70.2\%) & 1.000 \\
\hline Child-Pugh class B & $16(6.7 \%)$ & $43(12.0 \%)$ & 0.032 & $15(6.3 \%)$ & $15(6.3 \%)$ & 1.000 \\
\hline AFP $>200 \mathrm{ng} / \mathrm{mL}$ & 138 (57.7\%) & 196 (54.9\%) & 0.494 & 75 (3I.5\%) & $86(36.1 \%)$ & 0.286 \\
\hline Maximal diameter $>5 \mathrm{~cm}$ & $150(62.8 \%)$ & $206(57.7 \%)$ & 0.217 & 149 (62.6\%) & 149 (62.6\%) & 1.000 \\
\hline Three or more tumor nodules & $35(14.6 \%)$ & $73(20.4 \%)$ & $0.07 I$ & $34(14.3 \%)$ & $34(14.3 \%)$ & 1.000 \\
\hline Bilobar disease & $26(10.9 \%)$ & $61(17.1 \%)$ & 0.035 & $26(10.9 \%)$ & $26(10.9 \%)$ & 1.000 \\
\hline Median survival time (months) & $45(0-100)$ & $36(0-100)$ & $<0.001$ & $45(0-100)$ & $36(0-96)$ & 0.002 \\
\hline
\end{tabular}

Abbreviations: AFP, alpha-fetoprotein; BCLC, Barcelona Clinic Liver Cancer; HBV, hepatitis B virus; HCV, hepatitis C virus; LRT, locoregional therapy; PSM, propensity score matching; SR, surgical resection.

\section{BCLC C}
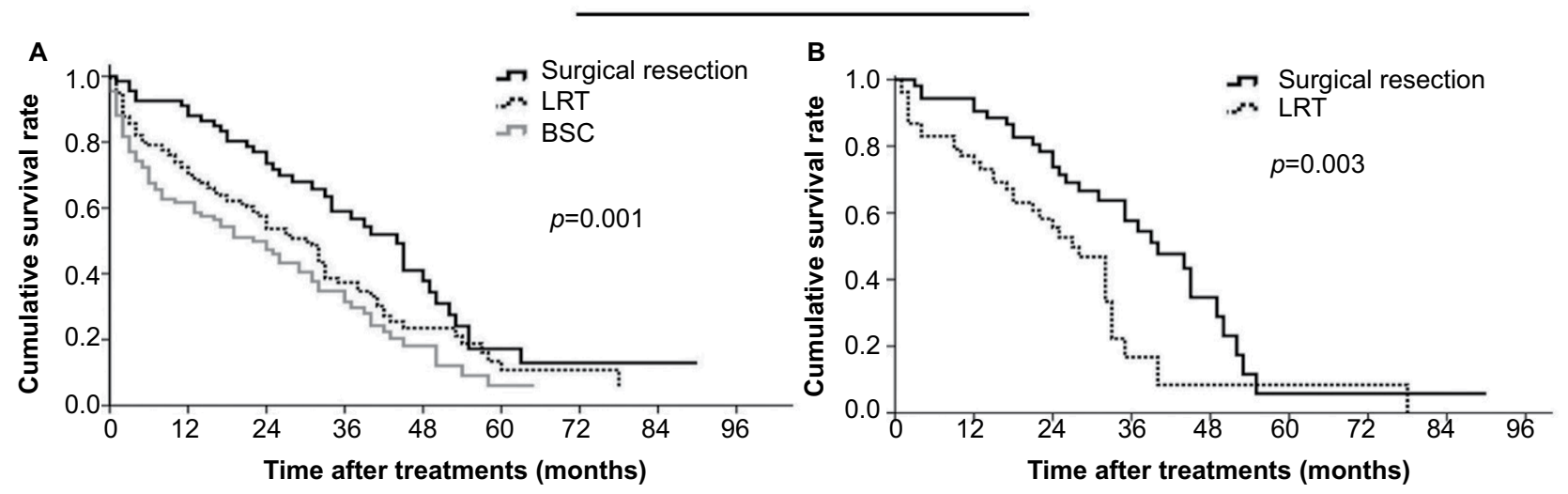

Figure 5 Overall survival of patients in BCLC stage $C$ after different treatments in unadjusted $(\mathbf{A})$ and adjusted (B) cohorts.

Abbreviations: BCLC, Barcelona Clinic Liver Cancer; BSC, best supportive care; LRT, locoregional therapy.

\section{Discussion}

HCC is a complex tumor with extremely heterogeneous biological behavior and multiple variables affecting treatment decision and outcome prediction. ${ }^{2}$ The BCLC staging system is now the most widely used for incorporating tumor burden, liver function and patient general condition. ${ }^{2,3}$ According to this staging system, surgical treatment should be reserved for patients with single lesion and normal bilirubin, but with no portal hypertension, while TACE and other palliative treatments are recommended as the first-line treatments to patients within BCLC stages B and C. ${ }^{2-4}$ Given that the BCLC staging system was developed $>10$ years ago based on a relatively small cohort of patients with predominantly $\mathrm{HCV}$-associated $\mathrm{HCC},{ }^{4}$ further refinement and amendments to the classification and treatment scheme should be considered, especially in HBV-endemic areas. ${ }^{16,17}$ As such, the current study aimed to evaluate the survival benefits of surgical resection vs other non-curative treatments in patients within different BCLC stages based on a large tertiary hospital database.

This study demonstrated an improved survival rate after surgical resection vs LRT across different BCLC stages in selected patients. It should not be overlooked that patients receiving surgery were often highly selected with younger age, better preoperative liver function, and lesser, smaller and more confined lesions. The PSM analysis was utilized to generate well-matched cohort, and it further confirmed that patients receiving surgical resection had better long-term survival than patients receiving LRT in BCLC stages B and C. 
Table 3 Characteristics of patients with BCLC stage C undergoing SR and LRT after PSM analysis

\begin{tabular}{|c|c|c|c|c|c|c|}
\hline \multirow[t]{2}{*}{ Variables } & \multicolumn{3}{|l|}{ Before PSM } & \multicolumn{3}{|l|}{ After PSM } \\
\hline & SR $(n=67)$ & LRT $(n=131)$ & $p$-value & $S R(n=53)$ & LRT $(n=53)$ & $p$-value \\
\hline Male gender & $53(79.1 \%)$ & $108(82.4 \%)$ & 0.569 & 42 (79.2\%) & $46(86.8 \%)$ & 0.458 \\
\hline Age (years) & $50.5(21-83)$ & $53(7-87)$ & 0.202 & $5 I(2 \mid-83)$ & $52(30-87)$ & 0.355 \\
\hline Smoking & 37 (55.2\%) & $70(53.4 \%)$ & 0.811 & 31 (58.5\%) & $26(49.1 \%)$ & 0.330 \\
\hline Alcohol abuse & 30 (44.8\%) & $50(38.2 \%)$ & 0.370 & $24(45.3 \%)$ & $18(34.0 \%)$ & 0.233 \\
\hline Diabetes & $6(9.0 \%)$ & $9(6.9 \%)$ & 0.632 & $6(11.3 \%)$ & $2(3.8 \%)$ & 0.270 \\
\hline HBV positivity & $54(80.6 \%)$ & $102(77.9 \%)$ & 0.656 & 40 (75.5\%) & 36 (67.9\%) & 0.388 \\
\hline HCV positivity & $2(3.0 \%)$ & 7 (5.3\%) & $0.45 \mathrm{I}$ & $2(3.8 \%)$ & $2(3.8 \%)$ & 1.000 \\
\hline Liver cirrhosis & 47 (70.1\%) & $96(73.3 \%)$ & $0.64 I$ & $34(64.2 \%)$ & $28(52.8 \%)$ & 0.331 \\
\hline Child-Pugh class B & $5(7.5 \%)$ & $24(18.3 \%)$ & 0.041 & $5(9.4 \%)$ & $4(7.5 \%)$ & 1.000 \\
\hline AFP $>200 \mathrm{ng} / \mathrm{mL}$ & 48 (7I.6\%) & 95 (72.5\%) & 0.896 & 38 (7I.7\%) & 38 (7I.7\%) & 1.000 \\
\hline Maximal diameter $>5 \mathrm{~cm}$ & $34(50.7 \%)$ & 91 (69.5\%) & 0.010 & $33(62.3 \%)$ & 31 (58.5\%) & 0.845 \\
\hline Three or more tumor nodules & $4(6.0 \%)$ & $21(16.0 \%)$ & 0.045 & $4(7.5 \%)$ & $9(17.0 \%)$ & 0.236 \\
\hline Bilobar disease & $\mathrm{I}(1.5 \%)$ & $18(13.7 \%)$ & 0.004 & I (1.9\%) & 0 & 1.000 \\
\hline Macrovascular invasion & $52(77.6 \%)$ & $110(84.0 \%)$ & 0.272 & 45 (84.9\%) & $44(83.0 \%)$ & 1.000 \\
\hline Median survival time (months) & $42(I-90)$ & $24.5(0-78)$ & $<0.001$ & $39(3-90)$ & $27(I-78)$ & 0.003 \\
\hline
\end{tabular}

Abbreviations: AFP, alpha-fetoprotein; BCLC, Barcelona Clinic Liver Cancer; HBV, hepatitis B virus; HCV, hepatitis C virus; LRT, locoregional therapy; PSM, propensity score matching; SR, surgical resection.

Notably, $>75 \%$ of patients were classified as BCLC stages B and $\mathrm{C}$ in the cohort. With advancement of surgical techniques and perioperative care, certain BCLC treatment recommendations might not fit in the management of HCC nowadays, especially in intermediate and advanced BCLC stages of HCC. Consistent with this study, several other studies from both the east and west have suggested that surgical resection provides better long-term outcomes than other non-surgical treatments, even for patients with multiple lesions and major vascular invasion. ${ }^{9}, 18,19$ Therefore, in carefully selected patients with low surgical risk and preserved liver function, surgical resection is safe, and it is effective for patients with resectable multiple and vascular invaded HCCs.

However, surgical resection is not applicable to all patients in BCLC stages B and C, even when technically feasible. The great heterogeneous population included in the intermediate and advanced stages of BCLC urges establishment of selection criteria for surgical resection. In 2012, a panel of experts proposed four substages of BCLC stage B HCC based on Child-Pugh classification, Eastern Cooperative Oncology Group (ECOG) performance and "up-to-7" criteria. ${ }^{5}$ Yamakado et al subgrouped intermediate HCC based on Child-Pugh grade and the combination of four tumors and $7 \mathrm{~cm} .{ }^{20}$ However, these criteria are for patients who benefit mostly from TACE. Wada et al divided patients within intermediate stage into four substages based on tumor size and number. ${ }^{18}$ However, this study enrolled small number of patients and failed to compare the outcomes between surgical resection and other treatments. Therefore, more efforts are needed to elucidate the selection criteria for surgical resection of patients with intermediate stage HCC.
BCLC stage $\mathrm{C}$ is more advanced $\mathrm{HCC}$ associated with major vascular invasion and/or extrahepatic metastasis and, therefore, extremely poor outcomes. We introduced PSM analysis to minimize the potential confounding biases in surgery and LRT groups. And then, it was further confirmed that surgical resection provided longer survival in selected patients. Although sorafenib is the only recommended firstline treatment for advanced HCC in BCLC staging system, surgical resection, RFA and even TACE are no longer contraindicated and provide favorable outcomes. ${ }^{17,21}$ The Liver Cancer Study Group of Japan has proposed a macroscopic classification for HCC with portal vein tumor thrombus and found significant decrease in the survival rates from the groups of branches to main trunk portal vein invasion after surgical resection. ${ }^{22,23}$ Given the great heterogeneity of the patients within BCLC stage $\mathrm{C}$, proper selection criteria should be established to differentiate those who could benefit mostly from surgery from those who might not, even though the available evidence indicates that hepatectomyand thrombectomy-based multidisciplinary treatments are effective options for patients with major vascular invasion, including hepatic vein and inferior vena cava., 34,25

\section{Limitations}

The study had several limitations. First, as for all retrospective studies, there were undoubtedly some selection biases such as difference of patients between surgical and non-surgical groups, subjective treatment decision and disparate surgical techniques of the clinicians, frequently incomplete data and so on. Therefore, we conducted a PSM analysis to match the comparison groups with equal liver function and tumor 
burden. However, some influential factors might be difficult to document. Second, the great heterogeneity of patients in each BCLC stage might make outcome comparison following different treatments difficult. Third, patients within intermediate and advanced stages might accept multiple treatments sequentially, which would make direct comparison of every single treatment difficult. Therefore, the details and optimal timing of auxiliary treatments combined with hepatectomy remain an important topic for future research. Liver transplantation, as another curative treatment modality for strictly selected patients meeting Milan criteria, ${ }^{26}$ was not evaluated in the current study due to the small sample size available.

\section{Conclusion}

The data of this study demonstrated that surgical resection achieved significant better overall survival vs non-surgical treatments not only in early but also in intermediate and advanced BCLC stage HCCs. However, much more work should focus on the optimal selection criteria for surgical resection of patients among different BCLC stages. Undoubtedly, further amendment and refinement of the BCLC staging system taking into account the clinical practice findings and accommodating the progress of various treatment modalities are urgently needed.

\section{Acknowledgments}

This study was supported in part by the National Natural Science Foundation of China (No. 81372582), "New-Star" Young Scientists in Shaanxi Provincial Program (2014kjxx30), The Science and Technology Innovative Research Team of Shaanxi Province (No. 2014KCT-24) and The Clinical Research Award of the First Affiliated Hospital of Xi'an Jiaotong University, China (No. XJTU1AF-CRF-2017-004 and XJTU1AF-CRF-2015-018). The sponsor has no role in the study design, performance or data explanation.

\section{Disclosure}

The authors report no conflicts of interest in this work.

\section{References}

1. Zhang XF, Qi X, Meng B, et al. Prognosis evaluation in alpha-fetoprotein negative hepatocellular carcinoma after hepatectomy: comparison of five staging systems. Eur J Surg Oncol. 2010;36(8):718-724.

2. European Association For The Study OfThe L, European Organisation For R, Treatment OfC. EASL-EORTC clinical practice guidelines: management of hepatocellular carcinoma. J Hepatol. 2012;56(4):908-943.

3. Bruix J, Sherman M, American Association for the Study of Liver D. Management of hepatocellular carcinoma: an update. Hepatology. 2011;53(3):1020-1022.

4. Llovet JM, Bru C, Bruix J. Prognosis of hepatocellular carcinoma: the BCLC staging classification. Semin Liver Dis. 1999;19(3):329-338.
5. Bolondi L, Burroughs A, Dufour JF, et al. Heterogeneity of patients with intermediate (BCLC B) Hepatocellular Carcinoma: proposal for a subclassification to facilitate treatment decisions. Semin Liver Dis. 2012;32(4):348-359.

6. Forner A, Gilabert M, Bruix J, Raoul JL. Treatment of intermediate-stage hepatocellular carcinoma. Nat Rev Clin Oncol. 2014;11(9):525-535.

7. Chang YJ, Chung KP, Chang YJ, Chen LJ. Long-term survival of patients undergoing liver resection for very large hepatocellular carcinomas. $\mathrm{Br}$ J Surg. 2016;103(11):1513-1520.

8. Yin L, Li H, Li AJ, et al. Partial hepatectomy vs. transcatheter arterial chemoembolization for resectable multiple hepatocellular carcinoma beyond Milan Criteria: a RCT. J Hepatol. 2014;61(1):82-88.

9. Chang WT, Kao WY, Chau GY, et al. Hepatic resection can provide longterm survival of patients with non-early-stage hepatocellular carcinoma: extending the indication for resection? Surgery. 2012;152(5):809-820.

10. Liu PH, Lee YH, Hsia CY, et al. Surgical resection versus transarterial chemoembolization for hepatocellular carcinoma with portal vein tumor thrombosis: a propensity score analysis. Ann Surg Oncol. 2014;21(6):1825-1833.

11. Lee HS, Kim KM, Yoon JH, et al. Therapeutic efficacy of transcatheter arterial chemoembolization as compared with hepatic resection in hepatocellular carcinoma patients with compensated liver function in a hepatitis B virus-endemic area: a prospective cohort study. J Clin Oncol. 2002;20(22):4459-4465.

12. Osterreicher CH, Stickel F, Brenner DA. Genomics of liver fibrosis and cirrhosis. Semin Liver Dis. 2007;27(1):28-43.

13. Zhang XF, Liu XM, Wei T, et al. Clinical characteristics and outcome of hepatocellular carcinoma in children and adolescents. Pediatr Surg Int. 2013;29(8):763-770.

14. Cho Y, Sinn DH, Yu SJ, et al. Survival Analysis of Single Large (>5 $\mathrm{cm})$ Hepatocellular Carcinoma Patients: BCLC A versus B. PLoS One. 2016;11(11):e0165722.

15. Jung YK, Jung CH, Seo YS, et al. BCLC stage B is a better designation for single large hepatocellular carcinoma than BCLC stage A. $J$ Gastroenterol Hepatol. 2016;31(2):467-474.

16. Yau T, Tang VY, Yao TJ, Fan ST, Lo CM, Poon RT. Development of Hong Kong Liver Cancer staging system with treatment stratification for patients with hepatocellular carcinoma. Gastroenterology. 2014;146(7):1691-1700 e1693.

17. Liu PH, Hsia CY, Lee YH, et al. Surgical resection versus transarterial chemoembolization for BCLC stage C hepatocellular carcinoma. J Surg Oncol. 2015;111(4):404-409.

18. Wada H, Eguchi H, Noda T, et al. Selection criteria for hepatic resection in intermediate-stage (BCLC stage B) multiple hepatocellular carcinoma. Surgery. 2016;160(5):1227-1235.

19. Torzilli G, Belghiti J, Kokudo N, et al. A snapshot of the effective indications and results of surgery for hepatocellular carcinoma in tertiary referral centers: is it adherent to the EASL/AASLD recommendations?: an observational study of the HCC East-West study group. Ann Surg. 2013;257(5):929-937.

20. Yamakado K, Miyayama S, Hirota S, et al. Subgrouping of intermediate-stage (BCLC stage B) hepatocellular carcinoma based on tumor number and size and Child-Pugh grade correlated with prognosis after transarterial chemoembolization. Jpn J Radiol. 2014;32(5): 260-265.

21. Lencioni R, Chen XP, Dagher L, Venook AP. Treatment of intermediate/ advanced hepatocellular carcinoma in the clinic: how can outcomes be improved? Oncologist. 2010;15 Suppl 4:42-52.

22. Japan LCSGo. Japan LCSGo. The General Rules for the Clinical and Pathological Study of Primary Liver Cancer. 2nd English ed. Tokyo: Kanehara \& Co., Ltd.; 2003.

23. Kudo M, Izumi N, Ichida T, et al. Report of the 19th follow-up survey of primary liver cancer in Japan. Hepatol Res. 2016;46(5):372-390.

24. Wang Y, Yuan L, Ge RL, Sun Y, Wei G. Survival benefit of surgical treatment for hepatocellular carcinoma with inferior vena cava/right atrium tumor thrombus: results of a retrospective cohort study. Ann Surg Oncol. 2013;20(3):914-922. 
25. Katagiri S, Yamamoto M. Multidisciplinary treatments for hepatocellular carcinoma with major portal vein tumor thrombus. Surg Today. 2014;44(2):219-226.
26. Mazzaferro V, Regalia E, Doci R, et al. Liver transplantation for the treatment of small hepatocellular carcinomas in patients with cirrhosis. N Engl J Med. 1996;334:693-699.

Cancer Management and Research

\section{Publish your work in this journal}

Cancer Management and Research is an international, peer-reviewed open access journal focusing on cancer research and the optimal use of preventative and integrated treatment interventions to achieve improved outcomes, enhanced survival and quality of life for the cancer patient The manuscript management system is completely online and includes
Dovepress

a very quick and fair peer-review system, which is all easy to use. Visit $\mathrm{http}: / /$ www.dovepress.com/testimonials.php to read real quotes from published authors.

Submit your manuscript here: https://www.dovepress.com/cancer-management-and-research-journal 